\title{
Wuzhi capsule increased systemic exposure to methotrexate by inhibiting the expression of OAT1/3 and P-gp
}

\author{
Ran Fu, Xiao-Nan Wang, Cai-Hui Guo, Ying Li, Cong-Yang Ding, Ya-Jing Li, Zhan-Jun Dong \\ Department of Pharmacy, Hebei General Hospital, Shijiazhuang, China \\ Contributions: (I) Conception and design: R Fu, ZJ Dong; (II) Administrative support: ZJ Dong; (III) Provision of study materials or patients: R Fu, \\ XN Wang, ZJ Dong; (IV) Collection and assembly of data: R Fu, XN Wang, CH Guo, Y Li; (V) Data analysis and interpretation: R Fu, CY Ding, YJ \\ Li; (VI) Manuscript writing: All authors; (VII) Final approval of manuscript: All authors. \\ Correspondence to: Zhan-Jun Dong. Department of Pharmacy, Hebei General Hospital, Shijiazhuang 050051, China. Email: dzjlcyx@126.com.
}

\begin{abstract}
Background: Methotrexate (MTX) is an important anticancer agent and immunosuppressant with a narrow therapeutic window. Wuzhi capsule (WZC) is an extract of Schisandra which is widely used to treat liver diseases. Co-administration of MTX and WZC is common in the clinical setting, but research on the interaction between WZC and MTX is limited. This study aimed to investigate the effects of WZC on the pharmacokinetics of MTX in rats and to explore the role of membrane transport proteins OAT1/3 and P-gp in the interaction of these drugs.

Methods: Plasma MTX concentration was detected by ultra-performance liquid chromatography-tandem mass spectrometer (UPLC-MS/MS), and the messenger RNA (mRNA) and protein expression of OAT1/3 and P-gp was evaluated by reverse transcription quantitative polymerase chain reaction (RT-qPCR) and western blotting analyses, respectively.

Results: The study results revealed that co-administration of WZC decreased the $\mathrm{CL}_{\mathrm{z} / \mathrm{F}}$ and $\mathrm{V}_{\mathrm{z} / \mathrm{F}}$ of $M T X$, increased the $\mathrm{C}_{\max }$ and area under the curve [(AUC) $)_{0-24 \mathrm{~h}}$ ] of MTX, and inhibited OAT1/3 expression in the kidney and $\mathrm{P}$-gp expression in the small intestine.

Conclusions: The findings suggested that there is a drug interaction between WZC and MTX and that OAT1/3 in the kidney and P-gp in the small intestine may be the main targets mediating the drug interaction, and attention should be paid when they are used in combination.
\end{abstract}

Keywords: Wuzhi capsule (WZC); methotrexate (MTX); pharmacokinetic study; herb-drug interaction; Organic Anion Transporters (OATs); P-gp

Submitted Feb 21, 2021. Accepted for publication Apr 17, 2021.

doi: 10.21037/atm-21-1303

View this article at: http://dx.doi.org/10.21037/atm-21-1303

\section{Introduction}

Methotrexate (MTX), an immunosuppressant and anticancer agent, can affect DNA biosynthesis and inhibit cell proliferation by inhibiting the synthesis of tetrahydrofolic acid (1). In the clinical setting, it is widely used in the treatment of psoriasis, rheumatoid arthritis, acute lymphoblastic leukemia, breast cancer, and other diseases (2). Excretion of MTX happens mainly via urine as the unchanged form, and transporters are known to play major roles in the disposal of MTX $(3,4)$.
The drug MTX is the substrate of the transporters $\mathrm{P}$-gp and OAT1/3, which are important transporters in the absorption and excretion of MTX $(5,6)$. When MTX is used in combination with inhibitors or inducers of P-gp and OAT1/3, the plasma concentration of MTX can be increased or decreased, and transporter-based drug interactions may easily occur. Puerarin has been shown to inhibit P-gp expression in the small intestine and OAT1/3 expression in the kidney, resulting in increased systemic exposure to MTX (7). This drug interaction may threaten the life and health of patients, because MTX has a 
narrow therapeutic window and severe side effects, such as hepatotoxicity and nephrotoxicity $(6,8)$.

Wuzhi capsule (WZC) contains the ethanol extract of the active component of Schisandra chinensis. Its active ingredients are mainly lignans, including schisantherin A, schisandrin A, schizandrol B, and deoxyschizandrin $(9,10)$. Not only can WZC reduce transaminase level and promote the regeneration of liver cells, it can also effectively inhibit drug-induced damage to liver cells $(11,12)$. In the clinical setting, WZC is often used in combination with MTX to prevent and treat MTX-induced liver damage. In recent years, studies have shown that the active ingredients of $S$. chinensis or the related preparations not only significantly enhance the uptake of P-gp substrate by Caco-2 cells and inhibit its efflux, but also increase systemic exposure to digoxin, vincristine, FK506, and ginsenosides by inhibiting the expression of P-gp (13-15). Thus, we speculated that WZC may also inhibit the expression of P-gp. Moreover, co-administration of WZC may cause changes in the pharmacokinetics of MTX in vivo by affecting the expression of P-gp, but the effect of WZC on the pharmacokinetics of MTX has not been studied. Furthermore, OAT1/3 are other important transporters in the disposal process of MTX in vivo; however, there are limited studies on the effects of WZC on OAT1/3 expression.

Considering that MTX has a narrow therapeutic window, fluctuations in its blood concentration will limit its efficacy or cause serious side effects (16). In addition, the possibility of a drug interaction when WZC is combined with MTX necessitates the assessment of potential drug interactions between WZC and MTX. Thus, we conducted this study to investigate the effects of WZC on the pharmacokinetics of MTX. We also clarified the molecular mechanism by examining the messenger RNA (mRNA) and protein expression of OAT1/3 in the kidney and P-gp in the small intestine via reverse transcription quantitative polymerase chain reaction (RT-qPCR) and western blotting analyses to provide a reference for the clinical combination therapy of the 2 drugs and promote rational clinical use of MTX.

We present the following article in accordance with the ARRIVE reporting checklist (available at http://dx.doi. org/10.21037/atm-21-1303).

\section{Methods}

\section{Chemicals and reagents}

The WZC was provided by Hezheng Pharmaceutical
Company (Chengdu, Sichuan, China). Reference standard of MTX (purity >99\%) was purchased from the National Institutes for Food and Drug Control (Beijing, China). Aminopterin [internal standard (IS), purity $>98 \%$ ] was purchased from Shanghai Yuanye Bio-Technology Co., Ltd. (Shanghai, China). High performance liquid chromatography (HPLC)-grade acetonitrile, methanol, and formic acid were obtained from Thermo Fisher Scientific (Pittsburgh, PA, USA). Ultrapure water was obtained from Wahaha Group Co., Ltd. (Hangzhou, China). The TRNzol Universal Reagent, FastQuant RT Kit (with DNase), and SuperReal PreMix Plus (SYBR Green) were purchased from Tiangen Biotech Co., Ltd. (Beijing, China). Primers were purchased from Wuhan Servicebio Technology Co., Ltd. (Wuhan, China). A bicinchoninic acid (BCA) Protein Assay Kit was obtained from Wuhan Servicebio Technology Co., Ltd. Anti-OAT1/3 was purchased from Abcam (Shanghai, China). Anti-P-gp antibodies and anti-actin were purchased from Wuhan Servicebio Technology Co., Ltd.

\section{Animal experiments}

Male Sprague-Dawley rats weighing 220-250 g were provided by the Experimental Animal Center of Hebei Medical University (Shijiazhuang, Hebei, China). The animal experiment protocol was approved by the Hebei General Hospital Ethics Committee (Shijiazhuang, Hebei, China). All experimental procedures were fulfilled based on the FDA Guide for the Care and Use of Laboratory Animals (USA 1985).

All rats were fed in a breeding room with controlled environmental conditions $\left(25 \pm 2{ }^{\circ} \mathrm{C}, 50 \% \pm 10 \%\right.$ relative humidity, and 12-h dark-light cycle) and acclimatized for 5 days. Standard water and chow were provided ad libitum. The animals were fasted for $12 \mathrm{~h}$ before the experiment, during which time they were allowed only water.

\section{Instrumentation and conditions}

The chromatographic analysis was performed using a Shimadzu UPLC system (Shimadzu Corporation, Kyoto, Japan) and an AB Sciex 5500 triple quadrupole tandem mass spectrometer equipped with an electrospray ionization interface (Framingham, MA, USA). The sample was separated on a Waters Xbridge C18 column $(100 \times 2.1 \mathrm{~mm}$, i.d.; $2.5 \mu \mathrm{m}$ ) (Milford, MA, USA) at column temperature of $40{ }^{\circ} \mathrm{C}$ and eluted with a step gradient mobile phase consisting of $0.1 \%$ formic water (solvent $\mathrm{A}$ ) and acetonitrile 
Table 1 Primer sequences used in RT-qPCR

\begin{tabular}{lll}
\hline mRNA & Forward primer $\left(5^{\prime}-3^{\prime}\right)$ & Reverse primer $\left(5^{\prime}-3^{\prime}\right)$ \\
\hline Rats- $\beta$-actin & TGCTATGTTGCCCTAGACTTCG & GTTGGCATAGAGGTCTTACGG \\
Rats-Mdr1a & TCTGGTATGGGACTTCCTTGGT & TCCTTGTATGTTGTCGGGTTTG \\
Rats-SIc22a6 & GTCTACTGCGTTTTCCGGCT & GGCTGACTCAATGAAGAACCAAG \\
Rats-SIc22a8 & CAGTCTTCCCAATGACACCCAG & GATCGACTGGGCCATCTCCT \\
\hline
\end{tabular}

RT-qPCR, reverse transcription-quantitative polymerase chain reaction.

(solvent B): 0-0.3 $\min , 5 \% \mathrm{~B} ; 0.3-2.5 \mathrm{~min}, 5-85 \% \mathrm{~B}$; 2.5-2.8 $\mathrm{min}, 85 \% \mathrm{~B} ; 2.8-3.5 \mathrm{~min}, 85-5 \% \mathrm{~B} ; 3.5-5 \mathrm{~min}, 5 \%$ B. The flow rate was $0.5 \mathrm{~mL} / \mathrm{min}$, and the injection volume was $5 \mu \mathrm{L}$. Mass spectrometric detection was performed with electrospray ionization in the positive ion multiple reaction monitoring mode. The transitions of the precursor ion and production of the analyte were $\mathrm{m} / \mathrm{z} 455.1 \rightarrow 308.2$ for MTX [declustering potential (DP), $100 \mathrm{~V}$; collision energy (CE), $25 \mathrm{~V}$ ] and $\mathrm{m} / \mathrm{z} 441.3 \rightarrow 294.2$ for aminopterin $(\mathrm{DP}, 116 \mathrm{~V}$; CE, $25 \mathrm{~V})$.

\section{In vivo pharmacokinetic experiment}

To evaluate the effects of WZC on the pharmacokinetics of MTX, 36 rats were divided randomly into 6 groups of 6 rats each as follows: Group I received a single dose of $0.5 \%$ sodium carboxymethyl cellulose (CMC-Na) via oral gavage and $2 \mathrm{mg} / \mathrm{kg}$ MTX (dissolved in $0.5 \% \mathrm{CMC}$ $\mathrm{Na}$ ) via oral gavage $20 \mathrm{~min}$ later; Group II-III received a single dose of WZC (dissolved in 0.5\% CMC-Na; 150 and $450 \mathrm{mg} / \mathrm{kg}$ for Group II and III, respectively) via oral gavage and $2 \mathrm{mg} / \mathrm{kg}$ MTX via oral gavage $20 \mathrm{~min}$ later; Group IV received multiple doses of $0.5 \% \mathrm{CMC}-\mathrm{Na}$ via oral gavage for 7 consecutive days and $2 \mathrm{mg} / \mathrm{kg}$ MTX via oral gavage on the 7th day, at $20 \mathrm{~min}$ after $\mathrm{CMC}-\mathrm{Na}$ administration; Group $\mathrm{V}$-VI received multiple doses of WZC (150 and $450 \mathrm{mg} / \mathrm{kg}$ ) via oral gavage for 7 consecutive days and $2 \mathrm{mg} / \mathrm{kg}$ MTX via oral gavage on the 7 th day, at 20 min after WZC administration. Blood samples were collected into a heparinized tube via the oculi choroidal vein before (at $0 \mathrm{~h}$ ) drug administration and at $0.083,0.17,0.33,0.67,1$, $1.33,1.67,2,2.33,3,5,7,9,12$, and $24 \mathrm{~h}$ after oral MTX administration. The blood samples were centrifuged at 3,500 rpm for $10 \mathrm{~min}$, and the supernatant was then collected and frozen at $-80{ }^{\circ} \mathrm{C}$ until analysis. To precipitate the proteins in the plasma samples, $50 \mu \mathrm{L}$ of plasma sample was mixed with $10 \mu \mathrm{L}$ of IS working solution $(200 \mathrm{ng} / \mathrm{mL})$ in a $1.5 \mathrm{~mL}$ centrifuge tube, and $150 \mu \mathrm{L}$ of methanol was added to the tube. The mixture was then vortex-mixed for $1 \mathrm{~min}$ and centrifuged at $12,000 \mathrm{rpm}$ or $10 \mathrm{~min}$. The supernatant was then added to UPLC sample vials.

\section{$R T-q P C R$}

The kidney and small intestine of rats were collected and homogenized in TRNzol Universal to extract the total RNA. Next, $2 \mu \mathrm{L}$ of the total RNA solution was taken and analyzed on a Thermo NanoDrop 2000c (Thermo Fisher Scientific, Inc., Waltham, MA, USA) by a 260/280 nm absorbance ratio to determine the RNA concentration and purity. Next, $2 \mu \mathrm{g}$ of the total RNA was used to synthesize first-strand cDNA using a FastKing RT Kit, and the remaining RNA solution was stored at $-80{ }^{\circ} \mathrm{C}$ for later use. We performed qPCR using SuperReal PreMix Plus on an ABI Prism TM 7500 Real-Time qPCR system (Thermo Fisher Scientific, Inc., Waltham, MA, USA). The final reaction system $(20 \mu \mathrm{L})$ was prepared according to the manufacturer's instructions and added to optical 96well reaction plates covered with plate sealers. Each tube contained $10 \mu \mathrm{L}$ of $2 \times$ SuperReal PreMix Plus, $0.6 \mu \mathrm{L}$ of forward primers, $0.6 \mu \mathrm{L}$ of reverse primers, $0.5 \mu \mathrm{L}$ of $50 \times$ ROX Reference Dye, $0.5 \mu \mathrm{L}$ of cDNA, and $7.8 \mu \mathrm{L}$ of $\mathrm{RNase}$-free $\mathrm{ddH}_{2} \mathrm{O}$. We used $\beta$-actin as an internal reference for normalization. The sequences of the primers used are listed in Table 1. The PCR cycling conditions were as follows: $95{ }^{\circ} \mathrm{C}$ for $15 \mathrm{~min}$, followed by 40 cycles of $95{ }^{\circ} \mathrm{C}$ for $10 \mathrm{~s}$ and $60{ }^{\circ} \mathrm{C}$ for $32 \mathrm{~s}$. The relative expression of mRNA was analyzed using the $2^{-\Delta \Delta \mathrm{CT}}$ method.

\section{Western blotting analysis}

Rat tissue samples were added to 10 times the volume of radio-immunoprecipitation (RIPA) Lysis Buffer and fully homogenized. The homogenate was centrifuged at $12,000 \mathrm{rpm}$ for $10 \mathrm{~min}$ after a $30 \mathrm{~min}$ ice bath to extract the total protein. A BCA Protein Assay Kit 
was used to determine the total protein concentration. Protein samples were loaded onto $10 \%$ sodium dodecyl sulphate-polyacrylamide gel at $120 \mathrm{~V}$ until the indicator bromophenol blue appeared. Next, the samples were transferred to a polyvinylidene fluoride (PVDF) membrane. The PVDF membrane was then blocked with $5 \%$ skimmed milk powder dissolved in tris-buffered saline with Tween 20 (TBST) solution in a shaker for $1 \mathrm{~h}$, followed by incubation with primary antibodies of OAT1 $(1: 1,000)$ (Abcam, Shanghai, China), OAT3 $(1: 1,000)$ (Abcam, Shanghai, China), P-gp $(1: 1,000)$ (Wuhan Servicebio Technology Co., Ltd., Wuhan, China), and ACTIN (1:1,000) (Wuhan Servicebio Technology Co., Ltd., Wuhan, China) overnight at $4{ }^{\circ} \mathrm{C}$. After that, the membrane was washed with TBST solution 3 times (for 5 min each time), incubated for $30 \mathrm{~min}$ at room temperature with a secondary antibody diluted 3,000 times in TBST, and washed with TBST solution again 3 times. Mixed electrochemiluminescence solution was added to the membrane for color development. AlphaEaseFC (Alpha Innotech, Shanghai, China) was used for gray level analysis. Then calculated the gray value ratio between the target strip and actin to normalized all target protein with actin. Each sample was repeated three times.

\section{Statistical analysis}

The pharmacokinetic parameters of MTX were analyzed using the DAS 2.1.1 software (Mathematical Pharmacology Professional Committee of China, Shanghai, China). All results were expressed as means \pm standard deviation (SD). Statistical analysis was performed using the SPSS 21.0 software (SPSS Inc., Chicago, IL, USA). Differences between groups were compared by $t$-test and nonparametric rank-sum test. Differences with $\mathrm{P}<0.05$ were considered statistically significant.

\section{Results}

\section{Effects of WZC on the Pharmacokinetics of MTX}

The mean plasma concentration-time curves of MTX after oral administration, with or without co-administration of WZC, are shown in Figure 1. The pharmacokinetic parameters were calculated using the non-compartmental method with the DAS 2.1.1 pharmacokinetic software, and the results are summarized in Tables 2 and 3.

The results of pharmacokinetic analysis showed that single-dose administration of $150 \mathrm{mg} / \mathrm{kg}$ WZC had no significant effect on the pharmacokinetic parameters of MTX. Following a single-dose administration of $450 \mathrm{mg} / \mathrm{kg}$ WZC, the $\mathrm{AUC}_{(0-24 \mathrm{~h})}$ of MTX increased significantly from $468.4 \pm 188.4$ to $1,024.8 \pm 173.6 \mu \mathrm{g} \cdot \mathrm{h} / \mathrm{L}(\mathrm{P}<0.01)$, the $\mathrm{C}_{\max }$ increased from $150.3 \pm 49.5$ to $269.3 \pm 32.9 \mathrm{ng} / \mathrm{mL}$ $(\mathrm{P}<0.01)$, the $\mathrm{V}_{z / \mathrm{F}}$ decreased from $50.2 \pm 22.5$ to $11.1 \pm 5.9 \mathrm{~L} / \mathrm{kg}(\mathrm{P}<0.01)$, and the $\mathrm{CL}_{z / \mathrm{F}}$ decreased from $4.54 \pm 1.76$ to $1.98 \pm 0.37 \mathrm{~L} / \mathrm{h} / \mathrm{kg}(\mathrm{P}<0.05)$. Moreover, multiple-dose administration of $150 \mathrm{mg} / \mathrm{kg}$ WZC led to a decrease in the $\mathrm{CL}_{\mathrm{z} / \mathrm{F}}$ of MTX from $4.65 \pm 1.12$ to $3.06 \pm 1.3 \mathrm{~L} / \mathrm{h} / \mathrm{kg}(\mathrm{P}<0.05)$. After multiple doses of $450 \mathrm{mg} / \mathrm{kg}$ WZC, the $\mathrm{AUC}_{(0-24 \mathrm{~h})}$ of MTX increased significantly from $440.2 \pm 96.1$ to $1,116 \pm 323.7 \mu \mathrm{g} \cdot \mathrm{h} / \mathrm{L}$ $(\mathrm{P}<0.01)$ and the $\mathrm{C}_{\max }$ increased from $162.3 \pm 11.2$ to $261 \pm 74.1 \mathrm{ng} / \mathrm{mL}(\mathrm{P}<0.05)$, whereas the $\mathrm{CL}_{z / \mathrm{F}}$ decreased from $4.65 \pm 1.12$ to $1.86 \pm 0.46 \mathrm{~L} / \mathrm{h} / \mathrm{kg}(\mathrm{P}<0.01)$.

\section{WZC inbibited OAT1 mRNA expression in the kidney and $P$-gp $m R N A$ expression in the small intestine}

Pharmacokinetic analysis results showed that WZC greatly affected the pharmacokinetics of MTX in vivo. Subsequently, we studied the mRNA expression of the transporters associated with the pharmacokinetics of MTX. The transporters were examined based on their tissue distributions; the expression of OAT1 and OAT3 in the kidneys as well as $\mathrm{P}$-gp in the small intestine was determined. The effect of WZC on the mRNA expression of the transporters is shown in Figure 2. The results of qPCR analysis revealed that $450 \mathrm{mg} / \mathrm{kg}$ WZC inhibited the mRNA expression of OAT1 in the kidney by $57 \%(\mathrm{P}<0.05)$ after a single dose and $67 \%(\mathrm{P}<0.01)$ after multiple doses, but had a marginal effect on the mRNA expression of OAT3. Furthermore, $450 \mathrm{mg} / \mathrm{kg}$ WZC reduced the mRNA expression of $\mathrm{P}$-gp in the small intestine by $54 \%(\mathrm{P}<0.05)$ after a single dose and by $64 \%(\mathrm{P}<0.01)$ after multiple doses.

\section{WZC inhibited OAT1/3 protein expression in the kidney and $P$-gp protein expression in the small intestine}

Based on the results of qPCR analysis, we measured the protein expression of OAT1/3 in the kidney and $\mathrm{P}$-gp in the small intestine. The effect of WZC on the protein expression of the transporters is shown in Figure 3. Western blotting results showed that the protein expression of OAT1 and OAT3 in the kidney was inhibited by $64 \%(\mathrm{P}<0.05)$ 
A
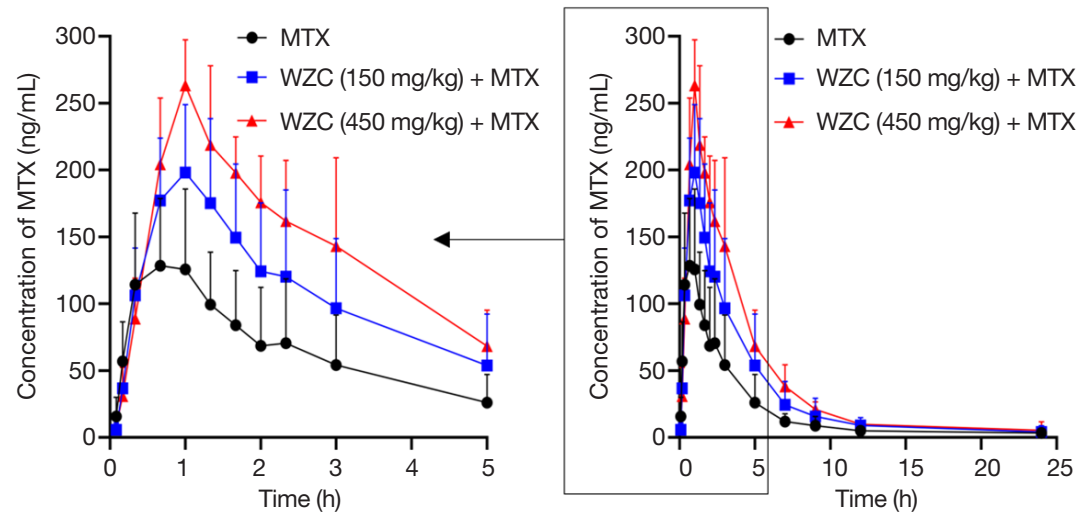

B
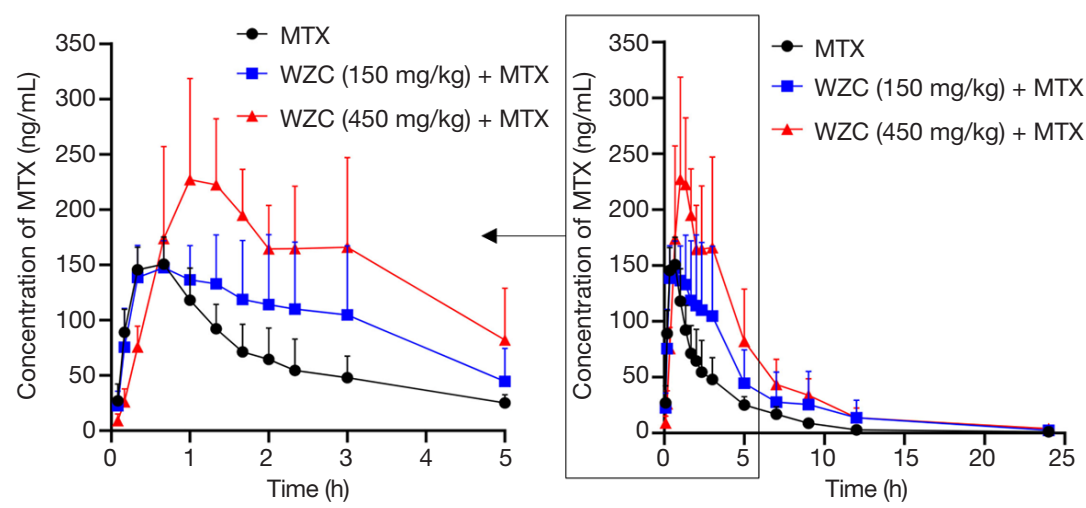

Figure 1 Mean plasma concentration-time curves of MTX in rats (6 rats in each group) after the oral administration of $2 \mathrm{mg} / \mathrm{kg}$ MTX with or without co-administration of (A) single-dose WZC and (B) multiple-dose WZC. Each point with a bar represents the mean \pm SD of 6 rats. MTX, methotrexate; WZC, Wuzhi capsule.

Table 2 Pharmacokinetic parameters of MTX in rats after intragastrical administration of MTX ( $2 \mathrm{mg} / \mathrm{kg}$; $\mathrm{n}=6$, mean \pm SD) with or without co-administration of single-dose WZC

\begin{tabular}{|c|c|c|c|}
\hline Parameter & MTX & WZC $(150 \mathrm{mg} / \mathrm{kg})+$ MTX & WZC $(450 \mathrm{mg} / \mathrm{kg})+\mathrm{MTX}$ \\
\hline $\mathrm{C}_{\max }(\mu \mathrm{g} / \mathrm{L})$ & $150.3 \pm 49.5$ & $202.8 \pm 49.9$ & $269.3 \pm 32.9^{\star *}$ \\
\hline$t_{1 / 2}(h)$ & $8.39 \pm 4.04$ & $6.67 \pm 6.24$ & $4.08 \pm 2.33$ \\
\hline $\operatorname{AUC}_{(0-24 h)}(\mu g \cdot h / L)$ & $468.4 \pm 188.4$ & $783.7 \pm 338.6$ & $1,024.8 \pm 173.6^{\star *}$ \\
\hline $\mathrm{V}_{\mathrm{z} / \mathrm{F}}(\mathrm{L} / \mathrm{kg})$ & $50.2 \pm 22.5$ & $29.3 \pm 35.1$ & $11.1 \pm 5.9^{\star *}$ \\
\hline
\end{tabular}

*, $\mathrm{P}<0.05 ;{ }^{* \star}, \mathrm{P}<0.01$ indicate significant differences from the MTX group. MTX, methotrexate; WZC, Wuzhi capsule; AUC, area under the curve.

and $57 \%(\mathrm{P}<0.05)$ after multiple-dose treatment with $150 \mathrm{mg} / \mathrm{kg}$ WZC and by $81 \%(\mathrm{P}<0.01)$ and $89 \%(\mathrm{P}<0.05)$ after multiple-dose treatment with $450 \mathrm{mg} / \mathrm{kg}$ WZC.
Moreover, $150 \mathrm{mg} / \mathrm{kg}$ WZC inhibited the protein expression of $\mathrm{P}$-gp in the small intestine by $37 \%(\mathrm{P}<0.05)$ and $45 \%(\mathrm{P}<0.05)$ after a single- and multiple-dose 
Table 3 Pharmacokinetic parameters of MTX in rats after intragastrical administration of MTX ( $\mathrm{mg} / \mathrm{kg}$; $\mathrm{n}=6$, mean \pm SD) with or without co-administration of multiple-dose WZC

\begin{tabular}{lccc}
\hline Parameter & MTX & WZC $(150 \mathrm{mg} / \mathrm{kg})+\mathrm{MTX}$ & WZC $(450 \mathrm{mg} / \mathrm{kg})+\mathrm{MTX}$ \\
\hline $\mathrm{T}_{\max }(\mathrm{h})$ & $0.5 \pm 0.18$ & $0.89 \pm 0.52$ & $1.39 \pm 0.83$ \\
$\mathrm{C}_{\max }(\mathrm{hg} / \mathrm{L})$ & $162.3 \pm 11.2$ & $170.8 \pm 35$ & $261 \pm 74.1^{\star}$ \\
$\mathrm{t}_{1 / 2}(\mathrm{~h})$ & $5.7 \pm 3.54$ & $5.34 \pm 1.02$ & $4.49 \pm 1.17$ \\
$\mathrm{AUC}_{(0-24 \mathrm{~h})}(\mu \mathrm{gg} \cdot \mathrm{h} / \mathrm{L})$ & $440.2 \pm 96.1$ & $781.6 \pm 466$ & $1,116 \pm 323.7^{\star *}$ \\
$\mathrm{C}_{\mathrm{LF}}(\mathrm{L} / \mathrm{h} / \mathrm{kg})$ & $4.65 \pm 1.12$ & $3.06 \pm 1.3^{*}$ & $1.86 \pm 0.46^{\star *}$ \\
$\mathrm{~V}_{z / \mathrm{F}}(\mathrm{L} / \mathrm{kg})$ & $40.5 \pm 34.4$ & $24.8 \pm 12.3$ & $12.6 \pm 5.9$ \\
\hline
\end{tabular}

${ }^{*}, \mathrm{P}<0.05 ;{ }^{* \star}, \mathrm{P}<0.01$ indicate significant differences from the MTX group. MTX, methotrexate; WZC, Wuzhi capsule; AUC, area under the curve.
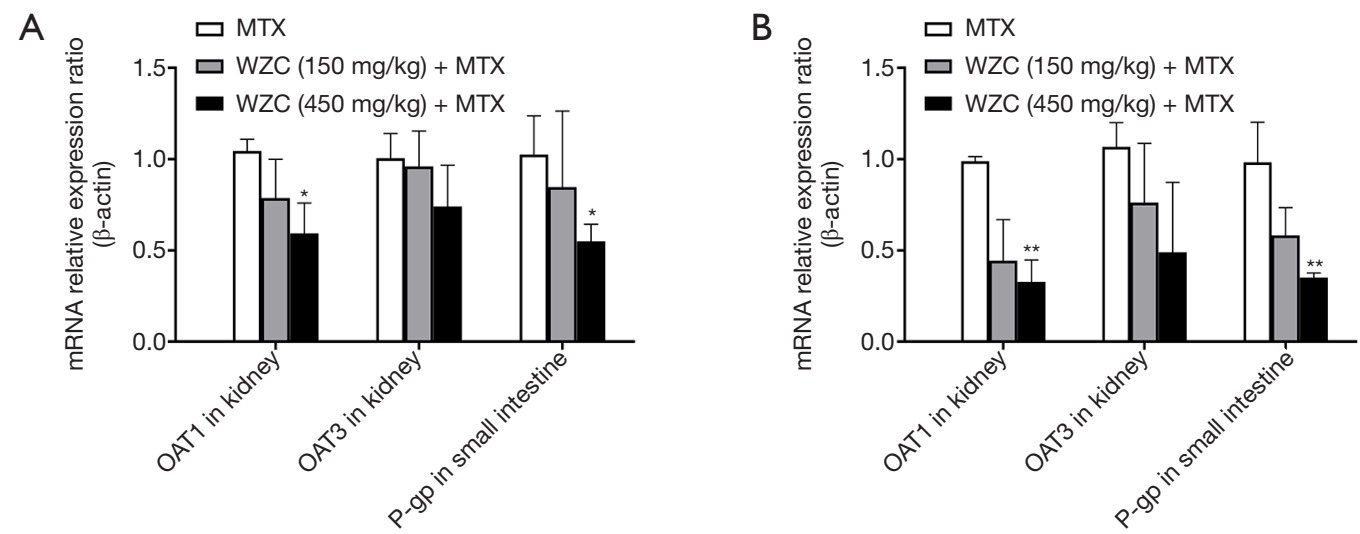

Figure 2 mRNA relative expression ratio of OAT1/3 in the kidney and P-gp in the small intestine at $24 \mathrm{~h}$ after the administration of MTX with or without co-administration of (A) single-dose WZC and (B) multiple-dose WZC. * $\mathrm{P}<0.05$; **, $\mathrm{P}<0.01$. MTX, methotrexate; WZC, Wuzhi capsule.

treatment, respectively. Furthermore, $450 \mathrm{mg} / \mathrm{kg}$ WZC inhibited $\mathrm{P}$-gp protein expression in the small intestine by 66\% $(\mathrm{P}<0.01)$ after a single dose and $83 \%(\mathrm{P}<0.01)$ after multiple doses.

\section{Discussion}

It has been widely acknowledged that WZC is an effective drug for the prevention and treatment of various kinds of drug-induced liver injury (17-19). The drug MTX is a chemotherapeutic agent that often causes adverse reactions, such as liver and kidney injury, during clinical treatment (8). However, although WZC is often used clinically in combination with MTX to prevent and treat MTX-induced liver injury, studies on the interaction between MTX and WZC are scarce. Considering that MTX has a narrow therapeutic window, and drug interactions associated with transporters are likely to occur when MTX is used in combination with other drugs (20-22), we conducted this study to investigate the effects of WZC on the pharmacokinetics of MTX.

We compared the effects of single- and multipledose treatment with WZC at different dosages on the pharmacokinetics of MTX. $2 \mathrm{mg} / \mathrm{kg}$ MTX and $150 \mathrm{mg} / \mathrm{kg}$ WZC were consistent with the clinical concentration of drug use. According to previous studies, high-dose WZC was set as $450 \mathrm{mg} / \mathrm{kg}$ (10). Pharmacokinetics results revealed that the combination of MTX and $450 \mathrm{mg} / \mathrm{kg}$ WZC reduced the $\mathrm{CL}_{\mathrm{z} / \mathrm{F}}$ and increased the $\mathrm{C}_{\max }$ and $\mathrm{AUC}_{(0-24 \mathrm{~h})}$ of MTX, regardless of whether WZC was administered in a single dose or multiple doses. It was shown that only the single dose of $450 \mathrm{mg} / \mathrm{kg}$ WZC reduced the $\mathrm{V}_{\mathrm{z} / \mathrm{F}}$ of MTX. 
A
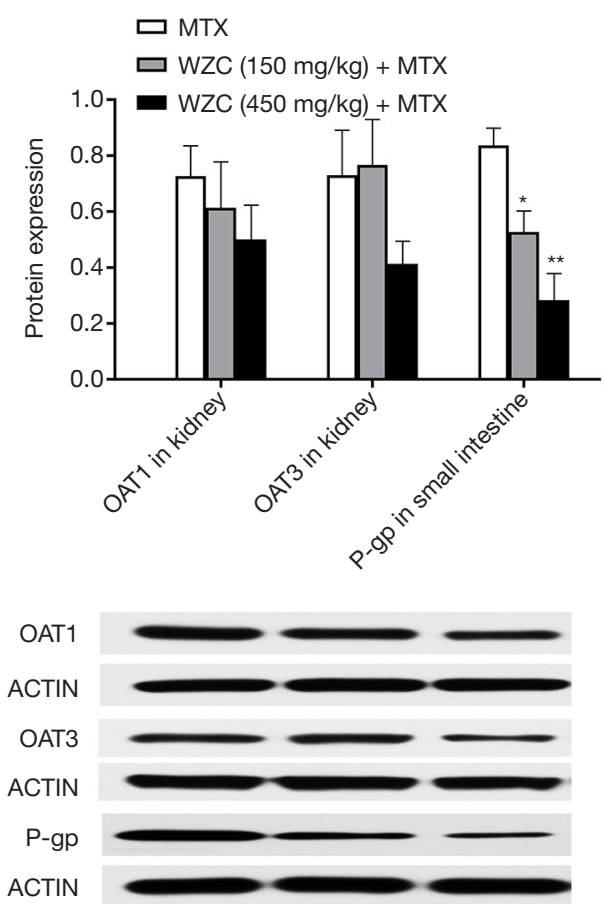

B
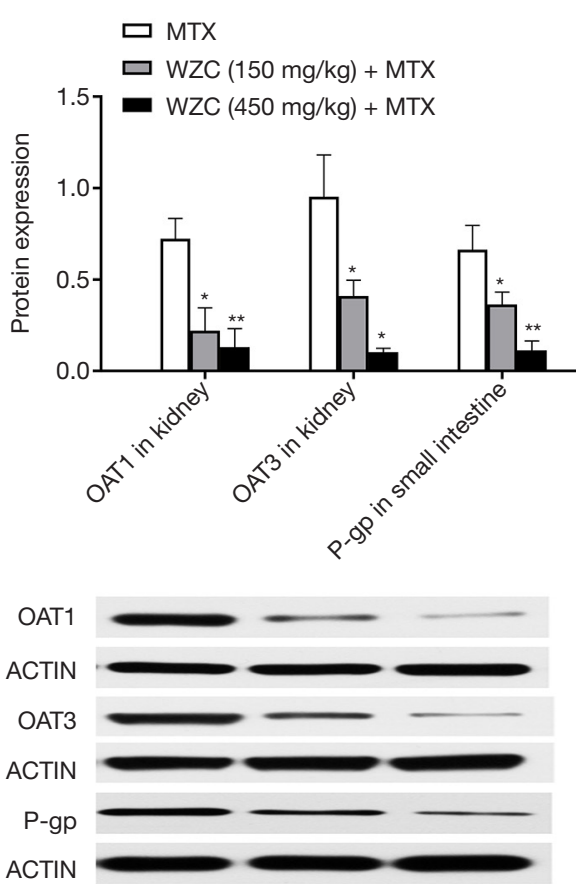

Figure 3 Protein expression ratio of OAT1 in the kidney and P-gp in the small intestine at $24 \mathrm{~h}$ after the administration of MTX with or without co-administration of (A) single-dose WZC and (B) multiple-dose WZC. *, P<0.05; **, P<0.01. MTX, methotrexate; WZC, Wuzhi capsule.

Though the difference in MTX plasma concentration between the control (CMC-Na-treated) group and the group treated with $150 \mathrm{mg} / \mathrm{kg}$ WZC was not significant, the trend of change in $\mathrm{CL}_{z / \mathrm{F}}, \mathrm{V}_{\mathrm{z} / \mathrm{F}}, \mathrm{C}_{\max }$, and $\mathrm{AUC}_{(0-24 \mathrm{~h})}$ was obviously the same as that in the group treated with $450 \mathrm{mg} / \mathrm{kg}$ WZC. This implied that although the impact of $150 \mathrm{mg} / \mathrm{kg}$ WZC has no statistically significant effect on the blood concentration of MTX, it still has the potential to increase the systemic exposure to MTX in vivo.

The transporters OAT1/3 belong to the Solute Carrier Family 22 (SLC22) $(23,24)$. They are distributed in many tissues, but mainly act in the kidney (25). In the kidney, they are expressed in the basal lateral membrane of renal tubular epithelial cells $(26,27)$, which promote the uptake of MTX from blood into renal tubular epithelial cells and mediate the urine excretion of MTX $(28,29)$. Previous studies have shown that puerarin can inhibit the renal excretion of MTX by inhibiting the expression of OAT1/3, resulting in increased plasma MTX level (7). In addition, bestatin inhibits the uptake of MTX in the kidney and increases blood MTX concentration by inhibiting the expression of OAT1/3 (20). In the present study, the mRNA and protein expression of OAT1 and the protein expression of OAT3 in the kidney was inhibited by WZC, which can lead to a reduction in the uptake of MTX in the kidney and might be an important reason for the decreased $\mathrm{CL}_{\mathrm{z} / \mathrm{F}}$ and increased systemic exposure to MTX.

The efflux transporter P-gp belongs to the adenosine triphosphate (ATP)-binding cassette transporter family (ABC) (27). It is mainly distributed in the small intestine and affects the reabsorption of MTX in the intestine $(30,31)$. Several studies have shown that the expression of P-gp greatly affects the plasma concentration of MTX, which may reduce the efficacy of MTX or promote serious side effects $(20,21,32)$. Thus, considering that many active ingredients of Schisandra are potent inhibitors of $\mathrm{P}$-gp $(13,15,33)$, we speculated that when used in combination with MTX, WZC may affect the pharmacokinetics of MTX by inhibiting the expression of P-gp. In our current study, the mRNA and protein expression of $\mathrm{P}$-gp was inhibited by WZC, which was consistent with the findings of previous research. The inhibition of $\mathrm{P}$-gp expression by WZC might cause a decrease in the non-renal clearance of MTX and an increase in the intestinal reabsorption of MTX. This may 
be another important reason for the increased systemic exposure to MTX.

Our study was the first to investigate the effect of WZC on the pharmacokinetics of MTX in rats. Although only high-dose WZC showed a statistically significant effect on the pharmacokinetics of MTX, the normal clinical dose of WZC $(150 \mathrm{mg} / \mathrm{kg})$ also resulted in a significant increase in systemic exposure to MTX and inhibited the protein expression of OAT1/3 and P-gp. Owing to the narrow therapeutic window of MTX and the large individual differences in the pharmacokinetics of MTX, it is still imperative to pay attention to drug interactions between WZC and MTX and to explore the safety of MTX when it is combined with WZC.

\section{Conclusions}

In conclusion, the effect of WZC on the pharmacokinetics of MTX is mediated by the action of multiple transporters in different tissues. Single- and multiple-dose administration of WZC had the same effect on the trends of MTX pharmacokinetics and transporter expression. As the dosage of WZC was increased, these effects became more obvious. In summary, we speculated that OAT $1 / 3$ in the kidney and $\mathrm{P}$-gp in the small intestine are the main targets mediating the drug interaction between WZC and MTX. The inhibition of OAT1/3 and P-gp expression by WZC led to a decrease in the $\mathrm{CL}_{\mathrm{z} / \mathrm{F}}$ and increases in the $\mathrm{C}_{\max }$ and $\mathrm{AUC}_{(0-24 \mathrm{~h})}$ of MTX. These results might help to provide a reference for the rational use of these two drugs in clinical practice. The interval of administration might be necessary when MTX and WZC are used together and further study is needed to explore the safety of MTX when it is combined with WZC.

\section{Acknowledgments}

We acknowledge the support of the Graduate School of Hebei Medical University, Shijiazhuang, Hebei, China, and Hebei General Hospital Clinical Research Center, Shijiazhuang, Hebei, China.

Funding: None.

\section{Footnote}

Reporting Checklist: The authors have completed the ARRIVE reporting checklist. Available at http://dx.doi. org/10.21037/atm-21-1303
Data Sharing Statement: Available at http://dx.doi. org/10.21037/atm-21-1303

Conflicts of Interest: All authors have completed the ICMJE uniform disclosure form (available at http://dx.doi. org/10.21037/atm-21-1303). The authors have no conflicts of interest to declare.

Ethical Statement: The authors are accountable for all aspects of the work in ensuring that questions related to the accuracy or integrity of any part of the work are appropriately investigated and resolved. The animal experiment protocol was approved by the Hebei General Hospital Ethics Committee (Shijiazhuang, Hebei, China). All experimental procedures were fulfilled based on the FDA Guide for the Care and Use of Laboratory Animals (USA 1985).

Open Access Statement: This is an Open Access article distributed in accordance with the Creative Commons Attribution-NonCommercial-NoDerivs 4.0 International License (CC BY-NC-ND 4.0), which permits the noncommercial replication and distribution of the article with the strict proviso that no changes or edits are made and the original work is properly cited (including links to both the formal publication through the relevant DOI and the license). See: https://creativecommons.org/licenses/by-nc-nd/4.0/.

\section{References}

1. Yu WJ, Huang DX, Liu S, et al. Polymeric Nanoscale Drug Carriers Mediate the Delivery of Methotrexate for Developing Therapeutic Interventions Against Cancer and Rheumatoid Arthritis. Front Oncol 2020;10:1734.

2. Shia CS, Juang SH, Tsai SY, et al. Interaction of rhubarb and methotrexate in rats: in vivo and ex vivo approaches. Am J Chin Med 2013;41:1427-38.

3. Yang $\mathrm{L}, \mathrm{Wu} \mathrm{H}$, de Winter BCM, et al. Pharmacokinetics and pharmacogenetics of high-dose methotrexate in Chinese adult patients with non-Hodgkin lymphoma: a population analysis. Cancer Chemother Pharmacol 2020;85:881-97.

4. Hu YH, Zhou L, Wang SS, et al. Methotrexate Disposition in Pediatric Patients with Acute Lymphoblastic Leukemia: What Have We Learnt From the Genetic Variants of Drug Transporters. Curr Pharm Des 2019;25:627-34.

5. Tang H, Wu YJ, Xiao F, et al. Regulation of CP-25 on $\mathrm{P}$-glycoprotein in synoviocytes of rats with adjuvant 
arthritis. Biomed Pharmacother 2019;119:109432.

6. Lin SP, Yu CP, Hou YC, et al. Transporter-mediated interaction of indican and methotrexate in rats. Food Drug Anal 2018;26:S133-40.

7. Liu Q, Wang C, Meng Q, et al. MDR1 and OAT1/OAT3 Mediate the Drug-Drug Interaction between Puerarin and Methotrexate. Pharm Res 2014;31:1120-32.

8. Valer JB, Curra M, Gabriel AF, et al. Oral mucositis in childhood cancer patients receiving high-dose methotrexate: Prevalence, relationship with other toxicities and methotrexate elimination. Int J Paediatr Dent 2021;31:238-46.

9. Zhang H, Bu F, Li L, et al. Prediction of Drug-Drug Interaction between Tacrolimus and Principal Ingredients of Wuzhi Capsule in Chinese Healthy Volunteers Using Physiologically-Based Pharmacokinetic Modelling. Basic Clin Pharmacol Toxicol 2018;122:331-40.

10. Wei H, Tao X, Di P, et al. Effects of traditional chinese medicine Wuzhi capsule on pharmacokinetics of tacrolimus in rats. Drug Metab Dispos 2013;41:1398-403.

11. Chen L, Xiong X, Hou X, et al.. Wuzhi capsule regulates chloroacetaldehyde pharmacokinetics behaviour and alleviates high-dose cyclophosphamide-induced nephrotoxicity and neurotoxicity in rats. Basic Clin Pharmacol Toxicol 2019;125:142-51.

12. Fan J, Chen L, Lu X, et al. The Pharmacokinetic Prediction of Cyclosporin A after Coadministration with Wuzhi Capsule. AAPS PharmSciTech 2019;20:247.

13. Qin XL, Chen X, Wang Y, et al. In vivo to in vitro effects of six bioactive lignans of Wuzhi tablet (Schisandra sphenanthera extract) on the CYP3A/P-glycoproteinmediated absorption and metabolism of tacrolimus. Drug Metab Dispos 2014;42:193-9.

14. Liang Y, Zhou Y, Zhang J, et al. Pharmacokinetic compatibility of ginsenosides and Schisandra Lignans in Shengmai-san: from the perspective of p-glycoprotein. PLoS One 2014; 9:e98717.

15. Liang $Y$, Zhou $Y$, Zhang J, et al. In vitro to in vivo evidence of the inhibitor characteristics of Schisandra lignans toward P-glycoprotein. Phytomedicine 2013;20:1030-8.

16. Karami F, Ranjbar S, Ghasemi Y, et al. Analytical methodologies for determination of methotrexate and its metabolites in pharmaceutical, biological and environmental samples. J Pharm Anal 2019;9:373-91.

17. Zhang W, Zhu YD, Zhang QY, et al. Research progress in application and mechanism of Schisandrae Chinensis Fructus for prevention and treatment of liver diseases. Zhongguo Zhong Yao Za Zhi 2020;45:3759-69.
18. Zhu P, Li J, Fu X, et al. Schisandra fruits for the management of drug-induced liver injury in China: A review. Phytomedicine 2019;59:152760.

19. Kang D, Shao Y, Zhu Z, et al. Systematically identifying the hepatoprotective ingredients of schisandra lignan extract from pharmacokinetic and pharmacodynamic perspectives. Phytomedicine 2019;53:182-192.

20. Zhu Y, Meng Q, Wang C, et al. Methotrexate-bestatin interaction: involvement of $\mathrm{P}$-glycoprotein and organic anion transporters in rats. Int J Pharm 2014;465:368-77.

21. Li Y, Deng S, Zetahao Y, et al. Smilax glabra Rhizoma affects the pharmacokinetics and tissue distribution of methotrexate by increasing the P-glycoprotein mRNA expression in rats after oral administration. Mol Med Rep 2017;16:7633-40.

22. Wang L, Ma L, Lin Y, et al. Leflunomide Increases Hepatic Exposure to Methotrexate and Its Metabolite by Differentially Regulating Multidrug ResistanceAssociated Protein Mrp2/3/4 Transporters via Peroxisome Proliferator-Activated Receptor alpha Activation. Mol Pharmacol 2018;93:563-74.

23. Nigam SK, Bush KT, Martovetsky G, et al. The organic anion transporter (OAT) family: a systems biology perspective. Physiol Rev 2015;95:83-123.

24. Dragojevic J, Mihaljevic I, Popovic M, et al. Zebrafish (Danio rerio) Oat1 and Oat3 transporters and their interaction with physiological compounds. Comp Biochem Physiol B Biochem Mol Biol 2019;236:110309.

25. Zhang J, Wang H, Fan Y, et al. Regulation of organic anion transporters: Role in physiology, pathophysiology, and drug elimination. Pharmacol Ther 2021;217:107647.

26. Antonescu IE, Karlgren M, Pedersen ML, et al. Acamprosate Is a Substrate of the Human Organic Anion Transporter (OAT) 1 without OAT3 Inhibitory Properties: Implications for Renal Acamprosate Secretion and DrugDrug Interactions. Pharmaceutics 2020;12:390.

27. Turkova A, Zdrazil B. Current Advances in Studying Clinically Relevant Transporters of the Solute Carrier (SLC) Family by Connecting Computational Modeling and Data Science. Comput Struct Biotechnol J 2019;17:390-405.

28. Uwai Y. Enantioselective Drug Recognition by Drug Transporters. Molecules 2018;23:3062.

29. Lozano E, Briz O, Macias R, et al. Genetic Heterogeneity of SLC22 Family of Transporters in Drug Disposition. Journal of Personalized Medicine 2018;8:14.

30. Wu YJ, Wang C, Wei W. The effects of DMARDs on 
Page 10 of 10

the expression and function of P-gp, MRPs, BCRP in the treatment of autoimmune diseases. Biomed Pharmacother 2018;105:870-8.

31. Jiang B, Yan L, Wu Q. ABCB1 (C1236T) Polymorphism Affects P-Glycoprotein-Mediated Transport of

Methotrexate, Doxorubicin, Actinomycin D, and

Etoposide. DNA and Cell Biology 2019;38:485-90.

32. Jia Y, Liu Z, Wang C, et al. P-gp, MRP2 and OAT1/
Fu et al. Effects of WZC on the pharmacokinetics of MTX

OAT3 mediate the drug-drug interaction between resveratrol and methotrexate. Toxicol Appl Pharmacol 2016;306:27-35.

33. Yoo HH, Lee M, Lee MW, et al. Effects of Schisandra lignans on P-glycoprotein-mediated drug efflux in human intestinal Caco-2. Planta Med 2007;73:444-50.

(English Language Editor: J. Jones)
Cite this article as: Fu R, Wang XN, Guo CH, Li Y, Ding CY, Li YJ, Dong ZJ. Wuzhi capsule increased systemic exposure to methotrexate by inhibiting the expression of OAT1/3 and P-gp. Ann Transl Med 2021;9(10):845. doi: 10.21037/atm-21-1303 